\title{
REMARKS ON LOCAL LIE ALGEBRAS OF PAIRS OF FUNCTIONS
}

\author{
JOSEF JANYŠKA
}

\begin{abstract}
We study local Lie algebras of pairs of functions which generate infinitesimal symmetries of almost-cosymplectic-contact structures of odd dimensional manifolds.
\end{abstract}

Keywords: Almost-cosymplectic-contact structure; almost-coPoissonJacobi structure; infinitesimal symmetry; Lie algebra.

Mathematics Subject Classification 2010: 53C15, 53B99, $17 \mathrm{~B} 66$.

\section{INTRODUCTION}

It is very well known, [10], that (1-dimensional) local Lie algebras of functions on a $(2 n+1)$-dimensional manifold $\boldsymbol{M}$ are in one-to-one correspondence with Jacobi structures on $\boldsymbol{M}$ given by a skew symmetric 2-vector field $\Lambda$ and a vector field $E$ such that

$$
[E, \Lambda]=0, \quad[\Lambda, \Lambda]=-2 E \wedge \Lambda,
$$

where [,] is the Schouten-Nijenhuis bracket (see, for example, [16]) of skew symmetric multi-vector fields.

Then the Jacobi bracket

$$
[f, h]=\{f, h\}-f E . h+h E . f,
$$

where $\{f, h\}=\Lambda(d f, d h)$ is the Poisson bracket, defines on the sheaf of functions $C^{\infty}(\boldsymbol{M})$ the structure of a local (Jacobi) Lie algebra.

Let us assume the subsheaf $C_{E}^{\infty}(\boldsymbol{M})$ of functions constant on integral curves of the vector field $E$, i.e. such that $E . f=0$ (conserved functions in the terminology of [9]). Then the restriction of the above Jacobi bracket defines the Lie subalgebra $\left(C_{E}^{\infty}(\boldsymbol{M}) ;\{\},\right) \subset\left(C^{\infty}(\boldsymbol{M}) ;[],\right)$ of conserved functions. Really, if $f, h \in C_{E}^{\infty}(\boldsymbol{M})$ then

$$
E .\{f, h\}=\{E . f, h\}+\{f, E . h\}=0 .
$$

Moreover, the Hamilton-Jacobi lift

$$
X_{f}=d f^{\sharp}-f E
$$

Supported by the grant GA ČR 14-02476S. 
of a function $f \in C_{E}^{\infty}(\boldsymbol{M})$ is an infinitesimal symmetry of $(E, \Lambda)$, i.e. $L_{X_{f}} E=\left[X_{f}, E\right]=0$ and $L_{X_{f}} \Lambda=\left[X_{f}, \Lambda\right]=0$. In what follows we shall use the notation $\Lambda^{\sharp}(\alpha):=\alpha^{\sharp}=i_{\alpha} \Lambda$ for any 1 -form $\alpha$.

If the pair $(E, \Lambda)$ is regular (transitive in the terminology of [10]), i.e. $E \wedge \Lambda^{n} \not \equiv 0$, then there exist the unique 1 -form $\omega$ such that $i_{E} \omega=1$ and $i_{\omega} \Lambda=0$. Moreover, $\omega$ is a contact form, i.e. $\omega \wedge d \omega^{n} \not \equiv 0$ and $E$ is the Reeb vector field of the contact structure $(\omega, \Omega=d \omega)$, i.e. $i_{E} \Omega=0$. The pairs $(\omega, \Omega)$ and $(E, \Lambda)$ are said to be mutually dual. It is easy to see that the Hamilton-Jacobi lift of a conserved function is an infinitesimal symmetry of the contact pair $(\omega, \Omega)$, i.e. $L_{X_{f}} \omega=0$ and $L_{X_{f}} \Omega=0$. The Hamilton-Jacobi lift of conserved functions is a Lie algebra homomorphism from the Lie algebra $\left(C_{E}^{\infty}(\boldsymbol{M}),\{\},\right)$ to the Lie algebra $(\mathcal{L}(\omega, \Omega),[],) \subset(\mathcal{X}(\boldsymbol{M}),[]$,$) of infinitesimal symmetries of the contact$ structure $(\omega, \Omega)$ (respective to the Lie algebra $(\mathcal{L}(E, \Lambda),[]$,$) of infini-$ tesimal symmetries of the Jacobi structure $(E, \Lambda))$. Here $(\mathcal{X}(\boldsymbol{M}),[]$, is the Lie algebra of vector fields.

In [8] dual contact and Jacobi structures were generalized in the following sense. An almost-cosymplectic-contact (regular) structure (pair) is given by a pair $(\omega, \Omega)$ such that

$$
d \Omega=0, \quad \omega \wedge \Omega^{n} \not \equiv 0 .
$$

According to [12] there exists a unique dual almost-coPoisson-Jacobi structure (pair) given by a pair $(E, \Lambda)$ such that

$$
\left(\Omega_{\mid \operatorname{Im}\left(\Lambda^{\sharp}\right)}^{b}\right)^{-1}=\Lambda_{\mid \operatorname{Im}\left(\Omega^{b}\right)}^{\sharp}, \quad i_{E} \omega=1, \quad i_{E} \Omega=0, \quad i_{\omega} \Lambda=0,
$$

where $\Omega^{b}: T \boldsymbol{M} \rightarrow T^{*} \boldsymbol{M}$ is given by $\Omega^{b}(X):=X^{b}=i_{X} \Omega$. Then (see [8])

$$
[E, \Lambda]=-E \wedge \Lambda^{\sharp}\left(L_{E} \omega\right), \quad[\Lambda, \Lambda]=2 E \wedge\left(\Lambda^{\sharp} \otimes \Lambda^{\sharp}\right)(d \omega) .
$$

Remark 1.1. The almost-cosymplectic-contact pair and the dual almost-coPoisson-Jacobi pair generalize not only the contact and the dual Jacobi structures but also cosymplectic structures. Really, if $d \omega=0$ we obtain a cosymplectic pair (see, for example, [1]). The corresponding dual pair is coPoisson pair, [8], given by the pair $(E, \Lambda)$ such that $[E, \Lambda]=0,[\Lambda, \Lambda]=0$.

Remark 1.2. For cosymplectic and contact structures we have distinguished forms $\omega, \Omega$ and vector fields $E, \Lambda$. But for an almostcosymplectic-contact structure we have distinguished forms $\omega, L_{E} \omega, \Omega$, $d \omega$ and distinguished vector fields $E,\left(L_{E} \omega\right)^{\sharp}, \Lambda,\left(\Lambda^{\sharp} \otimes \Lambda^{\sharp}\right)(d \omega)$.

In what follows we assume an odd dimensional manifold $\boldsymbol{M}$ with a regular almost-cosymplectic-contact structure $(\omega, \Omega)$. We assume the 
dual (regular) almost-coPoisson-Jacobi structure $(E, \Lambda)$. Then we have $\operatorname{Ker}(\omega)=\operatorname{Im}\left(\Lambda^{\sharp}\right)$ and $\operatorname{Ker}(E)=\operatorname{Im}\left(\Omega^{b}\right)$ and we have the splitting

$$
T \boldsymbol{M}=\operatorname{Im}\left(\Lambda^{\sharp}\right) \oplus\langle E\rangle, \quad T^{*} \boldsymbol{M}=\operatorname{Im}\left(\Omega^{b}\right) \oplus\langle\omega\rangle,
$$

i.e. any vector field $X$ and any 1 -form $\beta$ can be decomposed as

$$
X=X_{(\alpha, h)}=\alpha^{\sharp}+h E, \quad \beta=\beta_{(Y, f)}=Y^{b}+f \omega,
$$

where $h, f \in C^{\infty}(\boldsymbol{M}), \alpha$ be a 1 -form and $Y$ be a vector field. Moreover, $h=\omega\left(X_{(\alpha, h)}\right)$ and $f=\beta_{(Y, f)}(E)$. Let us note that the splitting (1.1) is not defined uniquely, really $X_{\left(\alpha_{1}, h_{1}\right)}=X_{\left(\alpha_{2}, h_{2}\right)}$ if and only if $\alpha_{1}^{\sharp}=\alpha_{2}^{\sharp}$ and $h_{1}=h_{2}$, i.e. $\alpha_{1}^{\sharp}-\alpha_{2}^{\sharp}=0$ that means that $\alpha_{1}-\alpha_{2} \in\langle\omega\rangle$. Similarly $\beta_{\left(Y_{1}, f_{1}\right)}=\beta_{\left(Y_{2}, f_{2}\right)}$ if and only if $Y_{1}-Y_{2} \in\langle E\rangle$ and $f_{1}=f_{2}$.

The projections $p_{2}: T \boldsymbol{M} \rightarrow\langle E\rangle$ and $p_{1}: T \boldsymbol{M} \rightarrow \operatorname{Im}\left(\Lambda^{\sharp}\right)=\operatorname{Ker}(\omega)$ are given by $X \mapsto \omega(X) E$ and $X \mapsto X-\omega(X) E$. Equivalently, the projections $q_{2}: T^{*} \boldsymbol{M} \rightarrow\langle\omega\rangle$ and $q_{1}: T^{*} \boldsymbol{M} \rightarrow \operatorname{Im}\left(\Omega^{b}\right)=\operatorname{Ker}(E)$ are given by $\beta \mapsto \beta(E) \omega$ and $\beta \mapsto \beta-\beta(E) \omega$. Moreover, $\Lambda^{\sharp} \circ \Omega^{b}=p_{1}$ and $\Omega^{b} \circ \Lambda^{\sharp}=q_{1}$.

Remark 1.3. Let us consider the pair $(\omega, F)$ where $F=\Omega+d \omega$ is a closed 2 -form. Then the pair $(\omega, F)$ is not generally an almostcosymplectic-contact pair because it may not to be regular. We shall use the form $F$ later in Theorem 3.1 .

In [5] we have studied infinitesimal symmetries of tensor fields $\omega, \Omega$, $E, \Lambda$ generating the almost-cosymplectic-contact and the dual almostcoPoisson-Jacobi structures. Such symmetries are vector fields of the type $X_{(\alpha, h)}=\alpha^{\sharp}+h E$, where $\alpha$ and $h$ meet certain conditions and the fact that they generate infinitesimal symmetries of a tensor field defines a Lie algebra structure on some subsheaf of $\Omega^{1}(\boldsymbol{M}) \times C^{\infty}(\boldsymbol{M})$ of generators $(\alpha, h)$ of infinitesimal symmetries.

In this paper we shall study the situation where the 1 -form $\alpha$ is locally exact, i.e. $\alpha=d f$ for $f \in C^{\infty}(\boldsymbol{M})$. This leads to local Lie algebras of pairs of functions generating infinitesimal symmetries of some tensor fields. Such Lie algebras are 2-dimensional local Lie algebras in the sense of [10].

Generally, we can define local Lie algebra structure in $C^{\infty}(\boldsymbol{M}) \times$ $C^{\infty}(\boldsymbol{M})$ by a (double) bracket $\llbracket\left(f_{1}, h_{1}\right) ;\left(f_{2}, h_{2}\right) \rrbracket$ in $C^{\infty}(\boldsymbol{M}) \times C^{\infty}(\boldsymbol{M})$ satisfying the following conditions:

(1) it defines a Lie algebra structure in $C^{\infty}(\boldsymbol{M}) \times C^{\infty}(\boldsymbol{M})$ over $\mathbb{R}$,

(2) $\llbracket\left(f_{1}, h_{1}\right) ;\left(f_{2}, h_{2}\right) \rrbracket$ is continuous in $f_{i}$ and $h_{i}, i=1,2$,

(3) $\operatorname{supp} \llbracket\left(f_{1}, h_{1}\right) ;\left(f_{2}, h_{2}\right) \rrbracket \subset \operatorname{supp}\left(f_{1}, h_{1}\right) \cap \operatorname{supp}\left(f_{2}, h_{2}\right)$ for each $\left(f_{1}, h_{1}\right)$ and $\left(f_{2}, h_{2}\right)$, where $\operatorname{supp}\left(f_{i}, h_{i}\right)=\operatorname{supp}\left(f_{i}\right) \cap \operatorname{supp}\left(h_{i}\right)$. 
As an example we can consider two Jacobi pairs $\left(E_{i}, \Lambda_{i}\right), i=1,2$, and the corresponding Jacobi brackets $[,]_{i}$. Then the bracket

$$
\llbracket\left(f_{1}, h_{1}\right) ;\left(f_{2}, h_{2}\right) \rrbracket=\left(\left[f_{1}, f_{2}\right]_{1} ;\left[h_{1}, h_{2}\right]_{2}\right)
$$

defines the Lie algebra structure in $C^{\infty}(\boldsymbol{M}) \times C^{\infty}(\boldsymbol{M})$. As far as we know, the classification of local Lie algebras of pairs of functions is not known. In the paper we shall describe several subsheafs of $C^{\infty}(\boldsymbol{M}) \times C^{\infty}(\boldsymbol{M})$ with a Lie algebra structure given by the fact that pairs of functions generate infinitesimal symmetries of basic tensor fields $\omega, \Omega, E, \Lambda$ given by the almost-cosymplectic-contact and the dual almost-coPoisson-Jacobi structures.

All manifolds and mappings are assumed to be smooth.

\section{Local Lie ALGEBRAS OF GENERATORS OF INFINITESIMAL SYMMETRIES}

In this section we shall study local Lie algebras of pairs of functions which generate infinitesimal symmetries of basic tensor fields.

For two functions $f, h \in C^{\infty}(\boldsymbol{M})$ we define their pre-Hamiltonian lift to vector fields on $M$ by

$$
X_{(f, h)}=d f^{\sharp}+h E .
$$

Lemma 2.1. Let $\left(f_{i}, h_{i}\right) \in C^{\infty}(\boldsymbol{M}) \times C^{\infty}(\boldsymbol{M}), i=1,2$, be two pairs of functions on $\boldsymbol{M}$. Then

$$
\begin{aligned}
& {\left[X_{\left(f_{1}, h_{1}\right)}, X_{\left(f_{2}, h_{2}\right)}\right]=\left(d\left\{f_{1}, f_{2}\right\}+\left(E \cdot f_{1}\right)\left(L_{d f_{2}^{\sharp}}+h_{2} L_{E}\right) \omega\right.} \\
& \left.\quad-\left(E \cdot f_{2}\right)\left(L_{d f_{1}^{\sharp}}+h_{1} L_{E}\right) \omega-h_{2} d\left(E \cdot f_{1}\right)+h_{1} d\left(E \cdot f_{2}\right)\right)^{\sharp} \\
& \quad+\left(\left\{f_{1}, h_{2}\right\}-\left\{f_{2}, h_{1}\right\}-d \omega\left(d f_{1}^{\sharp}, d f_{2}^{\sharp}\right)\right. \\
& \left.\quad+h_{1}\left(E \cdot h_{2}+\Lambda\left(L_{E} \omega, d f_{2}\right)\right)-h_{2}\left(E \cdot h_{1}+\Lambda\left(L_{E} \omega, d f_{1}\right)\right)\right) E .
\end{aligned}
$$

Proof. We have (see [8])

$$
\begin{aligned}
{\left[E, d f^{\sharp}\right]=} & \left(d(E . f)-(E . f)\left(L_{E} \omega\right)\right)^{\sharp}+\Lambda\left(L_{E} \omega, d f\right) E, \\
{\left[d f^{\sharp}, d h^{\sharp}\right]=} & \left(d \Lambda(d f, d h)+(E . f)\left(i_{d h^{\sharp}} d \omega\right)\right. \\
& \left.-(E . h)\left(i_{d f \sharp} d \omega\right)\right)^{\sharp}-d \omega\left(d f^{\sharp}, d h^{\sharp}\right) E
\end{aligned}
$$

which implies (2.2).

It is easy to see that the vector field (2.2) is not generally the preHamiltonian lift of a pair of functions. It is so in the case when the projection $p_{1}: T \boldsymbol{M} \rightarrow \operatorname{Ker}(\omega)$ of $(2.2)$ is the $\Lambda^{\sharp}$-lift of the differential of a function. We shall describe several examples of subsheafs of $C^{\infty}(\boldsymbol{M}) \times C^{\infty}(\boldsymbol{M})$ such that the Lie bracket of two pre-Hamiltonian 
lifts of pairs of functions from the subsheaf is the pre-Hamiltonian lift of a pair from the subsheaf. All such subsheafs are given by local generators of infinitesimal symmetries of basic tensor fields.

\subsection{Infinitesimal symmetries of $\omega$ and $\Omega$ generated by pairs of functions.}

Theorem 2.2. The pre-Hamiltonian lift (2.1) is an infinitesimal symmetry of $\omega$ if and only if

$$
i_{d f \sharp} d \omega+h i_{E} d \omega+d h=0 .
$$

Proof. From $i_{E} \omega=1$ and $i_{d f \sharp} \omega=0$ we get

$$
L_{X_{(f, h)}} \omega=i_{d f \sharp} d \omega+h i_{E} d \omega+d h
$$

which proves Theorem 2.2

Let us denote by $\operatorname{LGen}(\omega) \subset C^{\infty}(\boldsymbol{M}) \times C^{\infty}(\boldsymbol{M})$ the subsheaf of pairs of functions $(f, h)$ on $\boldsymbol{M}$ which generate (locally) infinitesimal symmetries of $\omega$, i.e. satisfy the condition (2.5).

Theorem 2.3. The Lie bracket of the pre-Hamiltonian lifts of two pairs $\left(f_{i}, h_{i}\right) \in \operatorname{LGen}(\omega), i=1,2$, is the pre-Hamiltonian lift of a pair of functions from $\mathrm{LGen}(\omega)$.

Proof. If (2.5) is satisfied then $d h_{i}=-\left(i_{d f_{i}^{\sharp}}+h_{i} i_{E}\right) d \omega=-\left(L_{d f_{i}^{\sharp}}+\right.$ $\left.h_{i} L_{E}\right) \omega$ and $E \cdot h_{i}+\Lambda\left(L_{E} \omega, d f_{i}\right)=0$ which follows by evaluating (2.5) on $E$. Then we can rewrite (2.2) as

$$
\begin{gathered}
{\left[X_{\left(f_{1}, h_{1}\right)}, X_{\left(f_{2}, h_{2}\right)}\right]=\left(d\left(\left\{f_{1}, f_{2}\right\}-h_{2}\left(E \cdot f_{1}\right)+h_{1}\left(E \cdot f_{2}\right)\right)\right)^{\sharp}} \\
+\left(\left\{f_{1}, h_{2}\right\}-\left\{f_{2}, h_{1}\right\}-d \omega\left(d f_{1}^{\sharp}, d f_{2}^{\sharp}\right)\right) E
\end{gathered}
$$

which is the pre-Hamiltonian lift of the pair

$$
\begin{aligned}
\left(\left\{f_{1}, f_{2}\right\}-h_{2}\left(E \cdot f_{1}\right)+\right. & h_{1}\left(E \cdot f_{2}\right) ; \\
& \left.\left\{f_{1}, h_{2}\right\}-\left\{f_{2}, h_{1}\right\}-d \omega\left(d f_{1}^{\sharp}, d f_{2}^{\sharp}\right)\right) .
\end{aligned}
$$

The pair (2.7) is in $\operatorname{LGen}(\omega)$ which follows from the fact that, according to Theorem 2.2, the pre-Hamiltonian lifts (2.1) of pairs from LGen $(\omega)$ are infinitesimal symmetries of $\omega$. Then from $L_{[X, Y]}=L_{X} L_{Y}-L_{Y} L_{X}$ the Lie bracket (2.6) of two pre-Hamiltonian lifts of pairs from LGen $(\omega)$ is an infinitesimal symmetry of $\omega$ and, by Theorem 2.2. the pair (2.7) has to satisfy the condition (2.5), i.e. it is in $\operatorname{LGen}(\omega)$.

As a consequence we obtain the Lie bracket

$$
\begin{aligned}
\llbracket\left(f_{1}, h_{1}\right) ;\left(f_{2}, h_{2}\right) \rrbracket= & \left(\left\{f_{1}, f_{2}\right\}-h_{2}\left(E . f_{1}\right)+h_{1}\left(E \cdot f_{2}\right) ;\right. \\
& \left.\left\{f_{1}, h_{2}\right\}-\left\{f_{2}, h_{1}\right\}-d \omega\left(d f_{1}^{\sharp}, d f_{2}^{\sharp}\right)\right)
\end{aligned}
$$


which defines the local Lie algebra structure on LGen $(\omega)$. Moreover, the pre-Hamiltonian lift (2.1) is the Lie algebra homomorphism from the local Lie algebra $(\operatorname{LGen}(\omega) ; \llbracket, \rrbracket)$ to the Lie algebra $(\mathcal{L}(\omega) ;[],) \subset$ $(\mathcal{X}(\boldsymbol{M}) ;[]$,$) of infinitesimal symmetries of \omega$.

Now, we shall define a Lie algebra structure on the subsheaf $C_{E}^{\infty}(\boldsymbol{M}) \times$ $C^{\infty}(\boldsymbol{M})$ of pairs $(f, h)$ of functions, where $f$ is conserved.

Theorem 2.4. The Lie bracket of the pre-Hamiltonian lifts of two pairs $\left(f_{i}, h_{i}\right) \in C_{E}^{\infty}(\boldsymbol{M}) \times C^{\infty}(\boldsymbol{M}), i=1,2$, is the pre-Hamiltonian lift of a pair of functions from $C_{E}^{\infty}(\boldsymbol{M}) \times C^{\infty}(\boldsymbol{M})$.

Proof. For $E . f_{i}=0$ the vector field (2.2) has the expression

$$
\begin{aligned}
{\left[X_{\left(f_{1}, h_{1}\right)},\right.} & \left.X_{\left(f_{2}, h_{2}\right)}\right]=\left(d\left\{f_{1}, f_{2}\right\}\right)^{\sharp} \\
& +\left(\left\{f_{1}, h_{2}\right\}-\left\{f_{2}, h_{1}\right\}-d \omega\left(d f_{1}^{\sharp}, d f_{2}^{\sharp}\right)\right. \\
& \left.+h_{1}\left(E \cdot h_{2}+\Lambda\left(L_{E} \omega, d f_{2}\right)\right)-h_{2}\left(E \cdot h_{1}+\Lambda\left(L_{E} \omega, d f_{1}\right)\right)\right) E .
\end{aligned}
$$

This vector field is the pre-Hamiltonian lift (2.1) of the pair

$$
\begin{aligned}
\left(\left\{f_{1}, f_{2}\right\} ;\{\right. & \left.f_{1}, h_{2}\right\}-\left\{f_{2}, h_{1}\right\}-d \omega\left(d f_{1}^{\sharp}, d f_{2}^{\sharp}\right) \\
& \left.+h_{1}\left(E \cdot h_{2}+\Lambda\left(L_{E} \omega, d f_{2}\right)\right)-h_{2}\left(E \cdot h_{1}+\Lambda\left(L_{E} \omega, d f_{1}\right)\right)\right) .
\end{aligned}
$$

Moreover, the above pair is in $C_{E}^{\infty}(\boldsymbol{M}) \times C^{\infty}(\boldsymbol{M})$. Really,

$$
\begin{aligned}
E \cdot\left\{f_{1}, f_{2}\right\} & =\left\{E \cdot f_{1}, f_{2}\right\}+\left\{f_{1}, E \cdot f_{2}\right\}+i_{[E, \Lambda]}\left(d f_{1} \wedge d f_{2}\right) \\
& =-i_{E \wedge\left(L_{E} \omega\right)^{\sharp}}\left(d f_{1} \wedge d f_{2}\right)=0
\end{aligned}
$$

which proves Theorem 2.4

As a consequence, by observing $\Lambda\left(L_{E} \omega, d f\right)=\left(L_{E} \omega\right)^{\sharp} . f$, we obtained the Lie bracket

$$
\begin{aligned}
& \llbracket\left(f_{1}, h_{1}\right) ;\left(f_{2}, h_{2}\right) \rrbracket=\left(\left\{f_{1}, f_{2}\right\} ;\right. \\
& \left\{f_{1}, h_{2}\right\}-\left\{f_{2}, h_{1}\right\}-d \omega\left(d f_{1}^{\sharp}, d f_{2}^{\sharp}\right) \\
& \left.\left.\left.\quad+h_{1}\left(E \cdot h_{2}+\left(L_{E} \omega\right)^{\sharp} \cdot f_{2}\right)\right)-h_{2}\left(E \cdot h_{1}+\left(L_{E} \omega\right)^{\sharp} \cdot f_{1}\right)\right)\right)
\end{aligned}
$$

of pairs of functions from $C_{E}^{\infty}(\boldsymbol{M}) \times C^{\infty}(\boldsymbol{M})$ which defines the local Lie algebra structure on $C_{E}^{\infty}(\boldsymbol{M}) \times C^{\infty}(\boldsymbol{M})$.

In [5] it was proved that all infinitesimal symmetries of $\Omega$ are vector fields $X_{(\alpha, h)}=\alpha^{\sharp}+h E$, where $\alpha$ is a closed 1-form such that $\alpha(E)=0$. Hence, locally, $\alpha=d f$ for a function $f \in C_{E}^{\infty}(\boldsymbol{M})$ and any infinitesimal symmetry of $\Omega$ is locally the pre-Hamiltonian lift of a pair of functions from $C_{E}^{\infty}(\boldsymbol{M}) \times C^{\infty}(\boldsymbol{M})$. So the Lie algebra of local generators of infinitesimal symmetries of $\Omega$ is $(\operatorname{LGen}(\Omega), \llbracket, \rrbracket) \equiv\left(C_{E}^{\infty}(\boldsymbol{M}) \times C^{\infty}(\boldsymbol{M}), \mathbb{}, \mathbb{}, \mathbb{}\right)$ with the Lie bracket (2.9). The pre-Hamiltonian lift (2.1) is the Lie 
algebra homomorphism from the local Lie algebra $(\operatorname{LGen}(\Omega) ; \llbracket, \rrbracket)$ to the Lie algebra $(\mathcal{L}(\Omega) ;[],) \subset(\mathcal{X}(\boldsymbol{M}) ;[]$,$) of infinitesimal symmetries$ of $\Omega$.

Theorem 2.5. A vector field $X_{(f, h)}$ is an infinitesimal symmetry of the almost-cosymplectic-contact structure $(\omega, \Omega)$ if and only if $f \in C_{E}^{\infty}(\boldsymbol{M})$ and the condition (2.5) is satisfied.

Proof. It follows from Theorems 2.2 and 2.4 .

Lemma 2.6. A vector field $X_{(f, h)}$ is an infinitesimal symmetry of $(\omega, \Omega)$ if and only if the following conditions are satisfied

(1) $E . f=i_{E} d f=0$,

(2) $i_{E} d h+i_{E} i_{d f \sharp} d \omega=E \cdot h+\left(L_{E} \omega\right)^{\sharp} \cdot f=0$,

(3) $d \omega\left(d f^{\sharp}, \beta^{\sharp}\right)+h d \omega\left(E, \beta^{\sharp}\right)+d h\left(\beta^{\sharp}\right)=0$ for any 1-form $\beta$, especially, if we put $\beta=d g$ for a $g \in C^{\infty}(\boldsymbol{M})$, we get $\{g, h\}=d \omega\left(d g^{\sharp}, d f^{\sharp}\right)+h d \omega\left(d g^{\sharp}, E\right)$.

Proof. It is a consequence of Theorem 2.5] and Theorem 2.2 where we have evaluated the 1-form on the left hand side of (2.5) on $E$ (which gives the condition (2)) and on $\beta^{\sharp}$ for any 1-form $\beta$ (which gives the condition (3)).

We denote the sheaf of pairs of functions which locally generate infinitesimal symmetries of the almost-cosymplectic-contact structure $(\omega, \Omega)$ as $\operatorname{LGen}(\omega, \Omega)=\operatorname{LGen}(\omega) \cap \operatorname{LGen}(\Omega)$. The brackets (2.8) and (2.9) restricted for generators of infinitesimal symmetries of $(\omega, \Omega)$ give the equivalet expressions of the bracket

$$
\begin{aligned}
\llbracket\left(f_{1}, h_{1}\right) ; & \left(f_{2}, h_{2}\right) \rrbracket= \\
& =\left(\left\{f_{1}, f_{2}\right\} ;\left\{f_{1}, h_{2}\right\}-\left\{f_{2}, h_{1}\right\}-d \omega\left(d f_{1}^{\sharp}, d f_{2}^{\sharp}\right)\right) \\
& =\left(\left\{f_{1}, f_{2}\right\} ; d \omega\left(d f_{1}^{\sharp}, d f_{2}^{\sharp}\right)+h_{2}\left(L_{E} \omega\right)^{\sharp} \cdot f_{1}-h_{1}\left(L_{E} \omega\right)^{\sharp} \cdot f_{2}\right) \\
& =\left(\left\{f_{1}, f_{2}\right\} ; d \omega\left(d f_{1}^{\sharp}, d f_{2}^{\sharp}\right)+h_{1} E \cdot h_{2}-h_{2} E \cdot h_{1}\right)
\end{aligned}
$$

which defines the Lie algebra structure on $\operatorname{LGen}(\omega, \Omega)$.

Corollary 2.7. An infinitesimal symmetry of the cosymplectic structure $(\omega, \Omega)$ is of local type $X_{(f, h)}=d f^{\sharp}+h E$, where $f \in C_{E}^{\infty}(\boldsymbol{M})$ and $h$ is a constant.

Then the bracket (2.10) is reduced to

$$
\llbracket\left(f_{1}, h_{1}\right) ;\left(f_{2}, h_{2}\right) \rrbracket=\left(\left\{f_{1}, f_{2}\right\}, 0\right) .
$$

I.e. we obtain the subalgebra $\left(\operatorname{LGen}_{\text {cos }}(\omega, \Omega) ; \llbracket, \rrbracket\right)=\left(C_{E}^{\infty}(\boldsymbol{M}) ;\{\},\right) \oplus$ $(\mathbb{R} ;[]$,$) of local generators of infinitesimal symmetries of the cosymplec-$ tic structure. Here [,] is the trivial Lie bracket in $\mathbb{R}$. 
Proof. It follows from $d \omega=0$, then, from (2.5), $d h=0$.

Corollary 2.8. Any infinitesimal symmetry of the contact structure $(\omega, \Omega=d \omega)$ is of local type

$$
X_{(f,-f)}=d f^{\sharp}-f E,
$$

where $f \in C_{E}^{\infty}(\boldsymbol{M})$.

Then the bracket (2.10) is reduced to

$$
\llbracket\left(f_{1},-f_{1}\right) ;\left(f_{2},-f_{2}\right) \rrbracket=\left(\left\{f_{1}, f_{2}\right\},-\left\{f_{1}, f_{2}\right\}\right) .
$$

I.e. we obtain the subalgebra $\left(\operatorname{LGen}_{\text {con }}(\omega) ; \llbracket, \rrbracket\right) \subset(\operatorname{LGen}(\omega, \Omega) ; \llbracket, \rrbracket)$ of local generators of infinitesimal symmetries of the contact structure. Moreover, $\left(\operatorname{LGen}_{c o n}(\omega) ; \llbracket ; \rrbracket\right) \equiv\left(C_{E}^{\infty}(\boldsymbol{M}),\{\},\right)$.

Proof. It follows from $d \omega=\Omega, i_{E} \Omega=0$ and $i_{d f \sharp} \Omega=d f$. Then, from (2.5),$d f=-d h$.

\subsection{Infinitesimal symmetries of $E$ and $\Lambda$ generated by pairs of functions.}

Theorem 2.9. The pre-Hamiltonian lift (2.1) is an infinitesimal symmetry of the Reeb vector field $E$ if and only if

$$
\begin{aligned}
\left(d(E . f)-(E . f) L_{E} \omega\right)^{\sharp} & =0, \\
(E \cdot h)+\left(L_{E} \omega\right)^{\sharp} \cdot f & =0 .
\end{aligned}
$$

Proof. Let us assume that the pre-Hamiltonian lift of a pair $(f, h)$ is an infinitesimal symmetry of the Reeb vector field. Then from (2.3)

$$
0=\left[X_{(f, h)}, E\right]=-\left(d(E . f)-(E . f) L_{E} \omega\right)^{\sharp}-\left((E . h)+\left(L_{E} \omega\right)^{\sharp} . f\right) E
$$

which proves Theorem 2.9 .

If we have two pairs $\left(f_{i}, h_{i}\right)$ generating infinitesimal symmetries of $E$ then the Lie bracket of the corresponding pre-Hamiltonian lifts is

$$
\begin{aligned}
& {\left[X_{\left(f_{1}, h_{1}\right)}, X_{\left(f_{2}, h_{2}\right)}\right]=\left(d\left\{f_{1}, f_{2}\right\}+\left(E \cdot f_{1}\right) L_{d f_{2}^{\sharp}} \omega-\left(E \cdot f_{2}\right) L_{d f_{1}^{\sharp}} \omega\right)^{\sharp}} \\
& \quad+\left(\left\{f_{1}, h_{2}\right\}-\left\{f_{2}, h_{1}\right\}-d \omega\left(d f_{1}^{\sharp}, d f_{2}^{\sharp}\right)\right) E .
\end{aligned}
$$

The above vector field (2.13) is not generally the pre-Hamiltonian lift of a pair of functions. So, the sheaf of local generators of infinitesimal symmetries of $E$ is not a Lie algebra.

Theorem 2.10. The pre-Hamiltonian lift (2.1) is an infinitesimal symmetry of $\Lambda$, i.e. $L_{X_{(f, h)}} \Lambda=\left[X_{X_{(f, h)}}, \Lambda\right]=0$, if and only if the following condition is satisfied

$$
\left[d f^{\sharp}, \Lambda\right]-E \wedge\left(d h+h L_{E} \omega\right)^{\sharp}=0 .
$$


Proof. We have

$$
L_{X_{(f, h)}} \Lambda=\left[d f^{\sharp}, \Lambda\right]+[h E, \Lambda] .
$$

Theorem 2.10 follows from

$$
[h E, \Lambda]=h[E, \Lambda]-E \wedge d h^{\sharp}=-E \wedge\left(d h+h L_{E} \omega\right)^{\sharp} .
$$

Lemma 2.11. A vector field $X_{(f, h)}$ is an infinitesimal symmetry of $\Lambda$ if and only if the following conditions

$$
\begin{aligned}
E . f & =0, \\
d \omega\left(d f^{\sharp}, \beta^{\sharp}\right)+h d \omega\left(E, \beta^{\sharp}\right)+d h\left(\beta^{\sharp}\right) & =0,
\end{aligned}
$$

are satisfied for any 1 -form $\beta$.

Proof. It is sufficient to evaluate the 2-vector field on the left hand side of (2.14) on $\omega, \beta$ and $\beta, \gamma$, where $\beta, \gamma$ are closed 1-forms. We get

$$
i_{\left[d f^{\sharp}, \Lambda\right]-E \wedge\left(d h+h L_{E} \omega\right)^{\sharp}}(\omega \wedge \beta)=-\Lambda\left(i_{d f^{\sharp}} d \omega+h L_{E} \omega+d h, \beta\right)
$$

which vanishes if and only if (2.16) is satisfied.

On the other hand

$$
\begin{aligned}
i_{\left[d f^{\sharp}, \Lambda\right]-E \wedge\left(d h+h L_{E} \omega\right)^{\sharp}}(\beta \wedge \gamma)= & \\
= & \Lambda(d f, d \Lambda(\beta, \gamma))+\Lambda(\beta, d \Lambda(\gamma, d f))+\Lambda(\gamma, d \Lambda(d f, \beta)) \\
& -\beta(E) \Lambda\left(h L_{E} \omega+d h, \gamma\right)+\gamma(E) \Lambda\left(h L_{E} \omega+d h, \beta\right)
\end{aligned}
$$

which, by using (2.16), can be rewritten as

$$
\begin{aligned}
i_{\left[d f^{\sharp}, \Lambda\right]-} & E \wedge\left(d h+h L_{E} \omega\right)^{\sharp} \\
& +\beta \wedge \gamma)=-\frac{1}{2} i_{[\Lambda, \Lambda]}(d f \wedge \beta \wedge \gamma) \\
= & -i_{E \wedge\left(\Lambda^{\sharp} \otimes \Lambda^{\sharp}\right)(d \omega)}(d f \wedge \beta \wedge \gamma) \\
& +\beta(E) \Lambda\left(i_{d f^{\sharp}} d \omega, \gamma\right)-\gamma(E) \Lambda\left(i_{d f^{\sharp}} d \omega, \beta\right) \\
= & -d f(E) d \omega\left(\beta^{\sharp}, \gamma^{\sharp}\right)=-(E . f) d \omega\left(\beta^{\sharp}, \gamma^{\sharp}\right)
\end{aligned}
$$

which vanishes if and only if (2.15) is satisfied.

On the other hand if (2.15) and (2.16) are satisfied, then the 2-vector field $L_{X_{(f, h)}} \Lambda$ is the zero 2 -vector field.

So, local generators of infinitesimal symmetries of $\Lambda$ are from LGen $(\Omega)$ and generates also infinitesimal symmetries of $\Omega$, i.e. $\operatorname{LGen}(\Lambda) \subset$ $\operatorname{LGen}(\Omega)$ and the Lie algebra of local generators of infinitesimal symmetries of $\Lambda$ is the subalgebra $(\operatorname{LGen}(\Lambda) ; \llbracket, \rrbracket) \subset(\operatorname{LGen}(\Omega) ; \llbracket, \rrbracket)$ with the bracket

$$
\begin{aligned}
& \llbracket\left(f_{1}, h_{1}\right) ;\left(f_{2}, h_{2}\right) \rrbracket= \\
& =\left(\left\{f_{1}, f_{2}\right\} ; d \omega\left(d f_{1}^{\sharp}, d f_{2}^{\sharp}\right)+h_{1} E \cdot h_{2}-h_{2} E \cdot h_{1}\right)
\end{aligned}
$$


which we obtain from the bracket (2.9) restricted for functions satisfying (2.16).

Corollary 2.12. The Lie algebra of local generators of infinitesimal symmetries of the almost-coPoisson-Jacobi pair $(E, \Lambda)$ coincides with the local Lie algebra of generators of infinitesimal symmetries of $(\omega, \Omega)$.

Proof. From Theorem 2.9 and Lemma 2.11 the pre-Hamiltonian lift (2.1) is an infinitesimal symmetry of the pair $(E, \Lambda)$ if and only if the conditions (1), (2) and (3) of Lemma 2.6 are satisfied, i.e. if and only if it is an infinitesimal symmetry of the pair $(\omega, \Omega)$.

Remark 2.1. Let us consider subsheaves of $C^{\infty}(\boldsymbol{M}) \times C^{\infty}(\boldsymbol{M})$ given by conditions (1), (2) and (3) of Lemma 2.6. The subsheaf LGen $(\Omega)$ is given by the condition (1), the subsheaf $\operatorname{LGen}(\omega)$ is given by the conditions (2) and (3) and the subsheaf $\operatorname{LGen}(\Lambda)$ is given by the conditions (1) and (3). If we assume the subsheaf given by conditions (1) and (2) then, from Theorem 2.9, it is the subsheaf $\operatorname{LGen}(E, \Omega)$ of local generators of infinitesimal symmetries of the Reeb vector field $E$ and $\Omega$. The corresponding bracket will be

$$
\begin{aligned}
\llbracket\left(f_{1}, h_{1}\right) ; & \left(f_{2}, h_{2}\right) \rrbracket= \\
= & \left(\left\{f_{1}, f_{2}\right\} ;\left\{f_{1}, h_{2}\right\}-\left\{f_{2}, h_{1}\right\}-d \omega\left(d f_{1}^{\sharp}, d f_{2}^{\sharp}\right)\right) .
\end{aligned}
$$

So we obtain the following local Lie algebras of generators of infinites-

\begin{tabular}{|c|c|c|}
\hline 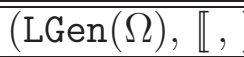 & $\overline{1})$ & 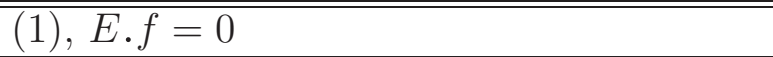 \\
\hline$(\operatorname{LGen}(\omega), \llbracket]$, & ) & $\begin{array}{l}\text { (2), } E \cdot h+\left(L_{E} \omega\right)^{\sharp} \cdot f=0 \\
(3), d \omega\left(d f^{\sharp}, \beta^{\sharp}\right)+h d \omega\left(E, \beta^{\sharp}\right)+d h\left(\beta^{\sharp}\right)=0\end{array}$ \\
\hline$(\operatorname{LGen}(\Lambda)$ & ) & (1) and (3) \\
\hline$(\operatorname{LGen}(E, \Omega)$ & $\llbracket, \rrbracket)$ & (1) and (2) \\
\hline $\begin{array}{l}(\operatorname{LGen}(\omega, \Omega), \\
\equiv(\operatorname{LGen}(E, \Lambda\end{array}$ & $\begin{array}{l}\left.\prod\right) \\
\llbracket, \rrbracket)\end{array}$ & $(1),(2)$ and $(3)$ \\
\hline
\end{tabular}
imal symmetries

Remark 2.2. In the Lie algebra $(\operatorname{LGen}(\Omega)$; $\llbracket$, $)$ we have the Abelian subalgebra formed by pairs of constant functions $\mathcal{K}(\boldsymbol{M})=\mathbb{R} \times \mathbb{R} \subset$ $\operatorname{LGen}(\Omega)$. The centralizer of the Lie subalgebra $\mathcal{K}(\boldsymbol{M})$ in LGen $(\Omega)$ is the Lie subalgebra $(\operatorname{LGen}(E, \Omega) ; \llbracket, \rrbracket) \subset(\operatorname{LGen}(\Omega) ; \llbracket, \rrbracket)$ of generators of infinitesimal symmetries of $E$ and $\Omega$.

Really, let $(c, k) \in \mathcal{K}(\boldsymbol{M})$ and $(f, h) \in \operatorname{LGen}(\Omega)$ such that

$$
(0 ; 0)=\llbracket(c, k) ;(f, h) \rrbracket=\left(0 ;-k\left(E . h+\left(L_{E} \omega\right)^{\sharp} \cdot f\right)\right) .
$$


Then, from Theorem 2.9, it follows that the pair $(f, h)$ generates an infinitesimal symmetry of $E$.

2.3. Multiplicative algebra $(\operatorname{LGen}(\Omega), \cdot)$. In Section 2.1 we have defined the local Lie algebra structure on $\operatorname{LGen}(\Omega)$. We define the multiplication in $\operatorname{LGen}(\Omega)$ by

$$
\left(f_{1}, h_{1}\right)\left(f_{2}, h_{2}\right)=\left(f_{1} f_{2}, f_{1} h_{2}+f_{2} h_{1}\right)
$$

which defines on $\operatorname{LGen}(\Omega)$ the structure of an associative commutative algebra with the unit $(1,0)$. Really it is easy to see that

$$
\begin{gathered}
\left(f_{1}, h_{1}\right)\left(f_{2}, h_{2}\right)=\left(f_{2}, h_{2}\right)\left(f_{1}, h_{1}\right), \\
\left(\left(f_{1}, h_{1}\right)\left(f_{2}, h_{2}\right)\right)\left(f_{3}, h_{3}\right)=\left(f_{1}, h_{1}\right)\left(\left(f_{2}, h_{2}\right)\left(f_{3}, h_{3}\right)\right), \\
(1,0)(f, h)=(f, h) .
\end{gathered}
$$

Lemma 2.13. We have

$$
X_{\left(f_{1} f_{2}, f_{1} h_{2}+f_{2} h_{1}\right)}=f_{1} X_{\left(f_{2}, h_{2}\right)}+f_{2} X_{\left(f_{1}, h_{1}\right)} .
$$

Proof. We have

$$
\begin{aligned}
X_{\left(f_{1} f_{2}, f_{1} h_{2}+f_{2} h_{1}\right)} & =d\left(f_{1} f_{2}\right)^{\sharp}+\left(f_{1} h_{2}+f_{2} h_{1}\right) E \\
& =f_{1}\left(d f_{2}^{\sharp}+h_{2} E\right)+f_{2}\left(d f_{1}^{\sharp}+h_{1} E\right) \\
& =f_{1} X_{\left(f_{2}, h_{2}\right)}+f_{2} X_{\left(f_{1}, h_{1}\right)} .
\end{aligned}
$$

From (2.9) it is easy to see that the bidifferential operator $D$ on LGen $(\Omega)$ given by

$$
D_{\left(f_{1}, h_{1}\right)}\left(f_{2}, h_{2}\right)=\llbracket\left(f_{1}, h_{1}\right) ;\left(f_{2}, h_{2}\right) \rrbracket
$$

is of order 1.

From the Jacobi identity we get

$$
D_{\llbracket\left(f_{1}, h_{1}\right) ;\left(f_{2}, h_{2}\right) \rrbracket}\left(f_{3}, h_{3}\right)=\left(D_{\left(f_{1}, h_{1}\right)} D_{\left(f_{2}, h_{2}\right)}-D_{\left(f_{2}, h_{2}\right)} D_{\left(f_{1}, h_{1}\right)}\right)\left(f_{3}, h_{3}\right)
$$

and

$$
\begin{aligned}
D_{\left(f_{1}, h_{1}\right)} \llbracket & \left(f_{2}, h_{2}\right) ;\left(f_{3}, h_{3}\right) \rrbracket= \\
& =\llbracket D_{\left(f_{1}, h_{1}\right)}\left(f_{2}, h_{2}\right) ;\left(f_{3}, h_{3} \rrbracket\right)+\llbracket\left(f_{2}, h_{2}\right) ; D_{\left(f_{1}, h_{1}\right)}\left(f_{3}, h_{3}\right) \rrbracket,
\end{aligned}
$$

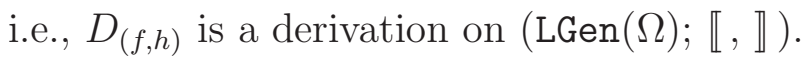

Theorem 2.14. The 1st order differential operator

$$
D_{(f, h)}: \operatorname{LGen}(\Omega) \rightarrow \operatorname{LGen}(\Omega)
$$


is a derivation on $(\operatorname{LGen}(\Omega), \cdot)$, i.e. for $\left(f_{i}, h_{i}\right) \in \operatorname{LGen}(\Omega), i=1,2,3$, we have

$$
\begin{aligned}
D_{\left(f_{1}, h_{1}\right)}\left(\left(f_{2}, h_{2}\right)\left(f_{3}, h_{3}\right)\right)=\left(f_{2}, h_{2}\right) D_{\left(f_{1}, h_{1}\right)} & \left(f_{3}, h_{3}\right) \\
& +\left(f_{3}, h_{3}\right) D_{\left(f_{1}, h_{1}\right)}\left(f_{2}, h_{2}\right) .
\end{aligned}
$$

Proof. From (2.9) we can prove

$$
\begin{aligned}
\llbracket\left(f_{1}, h_{1}\right) ;\left(f_{2}, h_{2}\right)\left(f_{3}, h_{3}\right) \rrbracket=\left(f_{2}, h_{2}\right) \llbracket & \left(f_{1}, h_{1}\right) ;\left(f_{3}, h_{3}\right) \rrbracket \\
& +\left(f_{3}, h_{3}\right) \llbracket\left(f_{1}, h_{1}\right) ;\left(f_{2}, h_{2}\right) \rrbracket
\end{aligned}
$$

which proves Theorem 2.14,

2.4. Lie derivation of pairs of functions. We define the Lie derivation of pairs of functions $(f, h) \in C^{\infty}(\boldsymbol{M}) \times C^{\infty}(\boldsymbol{M})$ given by a vector field $X$ on $\boldsymbol{M}$ by $L_{X}(f, h)=\left(L_{X} f, L_{X} h\right)=(X \cdot f, X . h)$. Generally $L_{X}$ is not an operator on $\operatorname{LGen}(\Omega)$.

Lemma 2.15. Let $X$ be a vector field on $M$ such that $L_{X} E=[X, E]=$ 0 . Then for $(f, h) \in \operatorname{LGen}(\Omega)$ the Lie derivation $L_{X}(f, h) \in \operatorname{LGen}(\Omega)$.

Proof. We have to prove that $L_{X} f \in C_{E}^{\infty}(\boldsymbol{M})$. But from

$$
0=L_{[X, E]} f=L_{X} L_{E} f-L_{E} L_{X} f=-E .\left(L_{X} f\right),
$$

hence $L_{X}(f, h) \in \operatorname{LGen}(\Omega)$.

Lemma 2.16. If a vector field $X$ is an infinitesimal symmetry of $E$ then $L_{X}$ is a derivation on $(\operatorname{LGen}(\Omega), \cdot)$, i.e.

$$
L_{X}\left(\left(f_{1}, h_{1}\right)\left(f_{2}, h_{2}\right)\right)=\left(L_{X} f_{1}, L_{X} h_{1}\right)\left(f_{2}, h_{2}\right)+\left(f_{1}, h_{1}\right)\left(L_{X} f_{2}, L_{X} h_{2}\right) .
$$

Proof. By Lemma 2.15 $L_{X}\left(\left(f_{1}, h_{1}\right)\left(f_{2}, h_{2}\right)\right) \in \operatorname{LGen}(\Omega)$ and

$$
\begin{aligned}
L_{X}\left(\left(f_{1}, h_{1}\right)\left(f_{2}, h_{2}\right)\right)=L_{X}\left(f_{1} f_{2}, f_{1} h_{2}+f_{2} h_{1}\right) & =\left(\left(L_{X} f_{1}\right) f_{2}+f_{1}\left(L_{X} f_{2}\right) ;\right. \\
& \left.\left(L_{X} f_{1}\right) h_{2}+f_{1}\left(L_{X} h_{2}\right)+\left(L_{X} f_{2}\right) h_{1}+f_{2}\left(L_{X} h_{1}\right)\right) \\
= & \left(L_{X} f_{1}, L_{X} h_{1}\right)\left(f_{2}, h_{2}\right)+\left(f_{1}, h_{1}\right)\left(L_{X} f_{2}, L_{X} h_{2}\right) .
\end{aligned}
$$

On the other hand it is easy to see that if $X$ is an infinitesimal symmetry of $E$, then $L_{X}$ is not a derivation on the Lie algebra $(\operatorname{LGen}(\Omega), \llbracket, \rrbracket)$. But we have

Theorem 2.17. Let $X$ be an infinitesimal symmetry of the almostcoPoisson-Jacobi structure $(E, \Lambda)$, then $X$ is a Lie derivation on the Lie algebra $(\operatorname{LGen}(\omega, \Omega) ; \llbracket, \rrbracket)$. 
Proof. $L_{X} E=0$ and $L_{X} \Lambda=0$ imply

$$
L_{X}\left\{f_{1}, f_{2}\right\}=\left\{L_{X} f_{1}, f_{2}\right\}+\left\{f_{1}, L_{X} f_{2}\right\}
$$

$L_{E} L_{X} \omega=0, L_{E} L_{X} f=L_{X} L_{E} f$ and $L_{X} d \omega=0$.

We have to prove that $\left(L_{X} f, L_{X} h\right) \in \operatorname{LGen}(\omega, \Omega)$ for any $(f, h) \in$ $\operatorname{LGen}(\omega, \Omega)$. First, from Lemma 2.15, $L_{X} f \in C_{E}^{\infty}(\boldsymbol{M})$. Further, we have to prove conditions (2) and (3) of Lemma 2.6 for the pair of functions $\left(L_{X} f, L_{X} h\right)$. The condition (2) can be expressed as

$$
0=d h(E)+\Lambda\left(L_{E} \omega, d f\right)
$$

If we apply $L_{X}$ on the above identity we get, from $L_{X} d f=d L_{X} f$,

$$
\begin{aligned}
0= & \left(L_{X} d h\right)(E)+d h\left(L_{X} E\right)+\left(L_{X} \Lambda\right)\left(L_{E} \omega, d f\right) \\
& +\Lambda\left(L_{X} L_{E} \omega, d f\right)+\Lambda\left(L_{E} \omega, L_{X} d f\right) \\
= & \left(d L_{X} h\right)(E)+\Lambda\left(L_{E} \omega, d L_{X} f\right)
\end{aligned}
$$

which is the condition (2) for the pair $\left(L_{X} f, L_{X} h\right)$.

Further, applying $L_{X}$ on (3) we get

$$
\begin{aligned}
0= & \left(L_{X} d \omega\right)\left(d f^{\sharp}, \beta^{\sharp}\right)+d \omega\left(L_{X} d f^{\sharp}, \beta^{\sharp}\right)+d \omega\left(d f^{\sharp}, L_{X} \beta^{\sharp}\right) \\
& +\left(L_{X} h\right) d \omega\left(E, \beta^{\sharp}\right)+h d \omega\left(L_{X} E, \beta^{\sharp}\right)+h d \omega\left(E, L_{X} \beta^{\sharp}\right) \\
& +\left(L_{X} d h\right)\left(\beta^{\sharp}\right)+d h\left(L_{X} \beta^{\sharp}\right) \\
= & d \omega\left(d\left(L_{X} f\right)^{\sharp}, \beta^{\sharp}\right)+\left(L_{X} h\right) d \omega\left(E, \beta^{\sharp}\right)+\left(d L_{X} h\right)\left(\beta^{\sharp}\right)
\end{aligned}
$$

which follows from $L_{X} d f^{\sharp}=d\left(L_{X} f\right)^{\sharp}$ (see [4], Lemma 2.15), and the condition (3) for the pair $\left(L_{X} f, L_{X} h\right)$ is satisfied. Hence $\left(L_{X} f, L_{X} h\right) \in$ $\operatorname{LGen}(\omega, \Omega)$.

Finally, we assume the bracket (2.10) in the form

$$
\llbracket\left(f_{1}, h_{1}\right) ;\left(f_{2}, h_{2}\right) \rrbracket=\left(\left\{f_{1}, f_{2}\right\} ; d \omega\left(d f_{1}^{\sharp}, d f_{2}^{\sharp}\right)+h_{1} E \cdot h_{2}-h_{2} E \cdot h_{1}\right) .
$$

Then

$$
\begin{aligned}
& L_{X} \llbracket\left(f_{1}, h_{1}\right) ;\left(f_{2}, h_{2}\right) \rrbracket=\left(\left\{L_{X} f_{1}, f_{2}\right\}+\left\{f_{1}, L_{X} f_{2}\right\} ;\right. \\
& \quad d \omega\left(L_{X} d f_{1}^{\sharp}, d f_{2}^{\sharp}\right)+d \omega\left(d f_{1}^{\sharp}, L_{X} d f_{2}^{\sharp}\right) \\
& \left.\quad+L_{X} h_{1} L_{E} h_{2}+h_{1} L_{X} L_{E} h_{2}-L_{X} h_{2} L_{E} h_{1}-h_{2} L_{X} L_{E} h_{1}\right) .
\end{aligned}
$$


On the other hand

$$
\begin{aligned}
\llbracket\left(L_{X} f_{1}, L_{X} h_{1}\right) ;( & \left.f_{2}, h_{2}\right) \rrbracket+\llbracket\left(f_{1}, h_{1}\right) ;\left(L_{X} f_{2}, L_{X} h_{2}\right) \rrbracket= \\
= & \left(\left\{L_{X} f_{1}, f_{2}\right\} ; d \omega\left(d\left(L_{X} f_{1}\right)^{\sharp}, d f_{2}^{\sharp}\right)\right. \\
& \left.\quad+L_{X} h_{1} L_{E} h_{2}-h_{2} L_{E} L_{X} h_{1}\right) \\
+ & \left(\left\{f_{1}, L_{X} f_{2}\right\} ; d \omega\left(d f_{1}^{\sharp}, d\left(L_{X} f_{2}\right)^{\sharp}\right)\right. \\
& \left.+h_{1} L_{E} L_{X} h_{2}-L_{X} h_{2} L_{E} h_{1}\right)
\end{aligned}
$$

and from $L_{X} d f^{\sharp}=d\left(L_{X} f\right)^{\sharp}$ we get

$$
\begin{aligned}
L_{X} \llbracket\left(f_{1}, h_{1}\right) ; & \left(f_{2}, h_{2}\right) \rrbracket= \\
& =\llbracket\left(L_{X} f_{1}, L_{X} h_{1}\right) ;\left(f_{2}, h_{2}\right) \rrbracket+\llbracket\left(f_{1}, h_{1}\right) ;\left(L_{X} f_{2}, L_{X} h_{2}\right) \rrbracket,
\end{aligned}
$$

i.e. $L_{X}$ is a derivation on the Lie algebra $(\operatorname{LGen}(\omega, \Omega)$; $\llbracket$, $)$.

Remark 2.3. We have

$$
\llbracket\left(f_{1}, h_{1}\right) ;\left(f_{2}, h_{2}\right) \rrbracket=\frac{1}{2}\left(L_{X_{\left(f_{1}, h_{1}\right)}}\left(f_{2}, h_{2}\right)-L_{X_{\left(f_{2}, h_{2}\right)}}\left(f_{1}, h_{1}\right)\right) .
$$

Really

$$
\begin{aligned}
L_{X_{\left(f_{1}, h_{1}\right)}}\left(f_{2}, h_{2}\right) & -L_{X_{\left(f_{2}, h_{2}\right)}}\left(f_{1}, h_{1}\right)= \\
& =\left(2\left\{f_{1}, f_{2}\right) ;\left\{f_{1}, h_{2}\right\}-\left\{f_{2}, h_{1}\right\}+h_{1} E . h_{2}-h_{2} E . h_{1}\right)
\end{aligned}
$$

and from (2) and (3) of Lemma 2.6] we have

$$
\left\{f_{1}, h_{2}\right\}-\left\{f_{2}, h_{1}\right\}=2 d \omega\left(d f_{1}^{\sharp}, d f_{2}^{\sharp}\right)+h_{1} E \cdot h_{2}-h_{2} E \cdot h_{1}
$$

which implies (2.17).

\section{LIE ALGEBROID AND INFINITESIMAL SYMMETRIES}

Let us assume a closed 2-form $F$ on $\boldsymbol{M}$ and the vector bundle $\boldsymbol{E}=$ $T \boldsymbol{M} \oplus_{\boldsymbol{M}} \mathbb{R} \rightarrow \boldsymbol{M}$. Then sections of $\boldsymbol{E}$ are pairs $(X, f)$ of vector fields on $\boldsymbol{M}$ and functions on $\boldsymbol{M}$. We define a bracket of sections of $\boldsymbol{E}$ by

$$
\llbracket\left(X_{1}, f_{1}\right) ;\left(X_{2}, f_{2}\right) \rrbracket_{F}=\left(\left[X_{1}, X_{2}\right] ; X_{1} . f_{2}-X_{2} . f_{1}+F\left(X_{1}, X_{2}\right)\right) .
$$

This bracket defines an F-Lie algebroid structure (see, for instance, [13]) on $\boldsymbol{E}$ with the anchor given by the projection on the first component.

Really, the bracket (3.1) is antisymmetric and from the closure of $F$ the Jacobi identity is satisfied. Moreover, for any $h \in C^{\infty}(\boldsymbol{M})$ we get

$$
\begin{aligned}
\llbracket\left(X_{1}, f_{1}\right) ; h\left(X_{2}, f_{2}\right) \rrbracket_{F} & =\llbracket\left(X_{1}, f_{1}\right) ;\left(h X_{2}, h f_{2}\right) \rrbracket_{F} \\
& =h \llbracket\left(X_{1}, f_{1}\right) ;\left(X_{2}, f_{2}\right) \rrbracket_{F}+\left(X_{1} . h\right)\left(X_{2}, f_{2}\right)
\end{aligned}
$$

and the Leibniz-type formula is satisfied. 
Now, let us assume the sheaf mapping from the local Lie algebra $(\operatorname{LGen}(\omega, \Omega) ; \llbracket, \rrbracket)$ to sections of the $F$-Lie algebroid given by pairs of vector fields on $\boldsymbol{M}$ and functions on $\boldsymbol{M}$ given by

$$
s:(f, h) \mapsto\left(X_{(f, h)}, f-h\right) .
$$

Theorem 3.1. For the closed 2-form

$$
F=\Omega+d \omega
$$

the sheaf mapping

$$
s: \operatorname{LGen}(\omega, \Omega) \rightarrow \mathcal{X}(\boldsymbol{M}) \times C^{\infty}(\boldsymbol{M})
$$

given by (3.2) is a Lie algebra morphism.

Proof. For $\left(f_{i}, h_{i}\right) \in \operatorname{LGen}(\omega, \Omega), i=1,2$, we have

$$
\begin{aligned}
& \llbracket\left(f_{1}, h_{1}\right) ;\left(f_{2}, h_{2}\right) \rrbracket=\left(\left\{f_{1}, f_{2}\right\} ;\left\{f_{1}, h_{2}\right\}-\left\{f_{2}, h_{1}\right\}-d \omega\left(d f_{1}^{\sharp}, d f_{2}^{\sharp}\right)\right) \\
& \mapsto\left(d\left\{f_{1}, f_{2}\right\}^{\sharp}+\right.\left(\left\{f_{1}, h_{2}\right\}-\left\{f_{2}, h_{1}\right\}-d \omega\left(d f_{1}^{\sharp}, d f_{2}^{\sharp}\right)\right) E ; \\
&\left.\left\{f_{1}, f_{2}\right\}-\left\{f_{1}, h_{2}\right\}+\left\{f_{2}, h_{1}\right\}+d \omega\left(d f_{1}^{\sharp}, d f_{2}^{\sharp}\right)\right) .
\end{aligned}
$$

On the other hand we have

$$
\begin{aligned}
& \llbracket \boldsymbol{s}\left(f_{1}, h_{1}\right) ; \boldsymbol{s}\left(f_{2}, h_{2}\right) \rrbracket_{F}= \\
& =\left(d\left\{f_{1}, f_{2}\right\}^{\sharp}+\left(\left\{f_{1}, h_{2}\right\}-\left\{f_{2}, h_{1}\right\}-d \omega\left(d f_{1}^{\sharp}, d f_{2}^{\sharp}\right)\right) E ;\right. \\
& \quad 2\left\{f_{1}, f_{2}\right\}-\left\{f_{1}, h_{2}\right\}+\left\{f_{2}, h_{1}\right\} \\
& \left.-h_{1} E . h_{2}+h_{2} E . h_{1}+F\left(d f_{1}^{\sharp}+h_{1} E, d f_{2}^{\sharp}+h_{2} E\right)\right) .
\end{aligned}
$$

The first parts of the above pairs are equal. The second parts are equal if and only if

$$
0=\left\{f_{1}, f_{2}\right\}-d \omega\left(d f_{1}^{\sharp}, d f_{2}^{\sharp}\right)-h_{1} E \cdot h_{2}+h_{2} E \cdot h_{1}+F\left(d f_{1}^{\sharp}+h_{1} E, d f_{2}^{\sharp}+h_{2} E\right)
$$

which can be rewritten, by using $\Omega\left(d f_{1}^{\sharp}, d f_{2}^{\sharp}\right)=-\Lambda\left(d f_{1}, d f_{2}\right)=-\left\{f_{1}, f_{2}\right\}$ (see [8]), as

$$
\begin{aligned}
0=-h_{1}\left(E \cdot h_{2}-F\left(E, d f_{2}^{\sharp}\right)\right)+h_{2}\left(E \cdot h_{1}\right. & \left.-F\left(E, d f_{1}^{\sharp}\right)\right) \\
& +(F-d \omega-\Omega)\left(d f_{1}^{\sharp}, d f_{2}^{\sharp}\right) .
\end{aligned}
$$

From $\Omega\left(E, d f_{i}^{\sharp}\right)=0, E \cdot h_{i}-d \omega\left(E, d f_{i}^{\sharp}\right)=0$ (see Lemma 2.6) it is equivalent with

$$
0=(F-d \omega-\Omega)\left(X_{\left(f_{1}, h_{1}\right)}, X_{\left(f_{2}, h_{2}\right)}\right)
$$

which is satisfied for $F=\Omega+d \omega$. 
Lemma 3.2. Let us consider the F-Lie algebroid given by the closed 2-form $F=\Omega+d \omega$. Let $\left(X_{i}, \breve{f}_{i}\right) \in \mathcal{X}(\boldsymbol{M}) \times C^{\infty}(\boldsymbol{M}), i=1,2$, be pairs such that $X_{i}$ are infinitesimal symmetries of $(\omega, \Omega)$ and $E . \breve{f}_{i}=$ $-E \cdot\left(\omega\left(X_{i}\right)\right)$. Then the bracket $\llbracket, \rrbracket_{F}$ of these pairs satisfy the same conditions.

Proof. $\left[X_{1}, X_{2}\right]$ is an infinitesimal symmetry of $(\omega, \Omega)$ so it is sufficient to prove that

$$
E \cdot\left(X_{1} \cdot \breve{f}_{2}-X_{2} \cdot \breve{f}_{1}+F\left(X_{1}, X_{2}\right)\right)=-E \cdot\left(\omega\left(\left[X_{1}, X_{2}\right]\right)\right)
$$

which can be rewritten as

$$
\begin{aligned}
X_{1} \cdot\left(E \cdot \breve{f}_{2}\right)-X_{2} \cdot\left(E \cdot \breve{f}_{1}\right)+E \cdot\left(F\left(X_{1}, X_{2}\right)\right)= \\
=-E \cdot\left(X_{1} \cdot\left(\omega\left(X_{2}\right)\right)-X_{2} \cdot\left(\omega\left(X_{1}\right)-d \omega\left(X_{1}, X_{2}\right)\right) .\right.
\end{aligned}
$$

From the condition $E . \breve{f}_{i}=-E \cdot\left(\omega\left(X_{i}\right)\right)$ this equation is satisfied if and only if

$$
E \cdot\left(F\left(X_{1}, X_{2}\right)\right)=E \cdot\left(d \omega\left(X_{1}, X_{2}\right)\right)
$$

which is satisfied for $F=\Omega+d \omega$ because of $L_{E} \Omega=0$ and $L_{E} X_{i}=$ $\left[E, X_{i}\right]=0$.

Theorem 3.3. Let us consider the F-Lie algebroid given by the closed 2-form $F=\Omega+d \omega$. Let $\left(X_{i}, \breve{f}_{i}\right) \in \mathcal{X}(\boldsymbol{M}) \times C^{\infty}(\boldsymbol{M}), i=1,2$, be pairs such that $X_{i}$ are infinitesimal symmetries of $(\omega, \Omega)$ and $E \cdot \breve{f}_{i}=$ $-E \cdot\left(\omega\left(X_{i}\right)\right)$. Then the sheaf mapping

$$
\boldsymbol{r}: \mathcal{X}(\boldsymbol{M}) \times C^{\infty}(\boldsymbol{M}) \rightarrow C^{\infty}(\boldsymbol{M}) \times C^{\infty}(\boldsymbol{M})
$$

given by

$$
\boldsymbol{r}:(X, \breve{f}) \mapsto(\omega(X)+\breve{f}, \omega(X))
$$

has values in $\operatorname{LGen}(\omega, \Omega)$ and it is a Lie algebra morphism inverse to $s$.

Proof. First we have to prove that the mapping (3.3) has values in $\operatorname{LGen}(\omega, \Omega)$. Let us assume that $X$ is an infinitesimal symmetry of $(\omega, \Omega)$. By Theorem $2.5 X=d f^{\sharp}+h E$, where E.f $=0$ and the condition (2.5) is satisfied. It is easy to see that $h=\omega(X)$. Then

$$
f=\omega(X)+\breve{f}
$$

is a conserved function. So we have

$$
X=d(\omega(X)+\breve{f})^{\sharp}+\omega(X) E
$$

and the pair $(\omega(X)+\breve{f}, \omega(X))$ is in $\operatorname{LGen}(\omega, \Omega)$. 
Now, we have

$$
\begin{aligned}
& \llbracket\left(X_{1}, \breve{f}_{1}\right) ;\left(X_{2}, \breve{f}_{2}\right) \rrbracket_{F} \mapsto \\
& \mapsto\left(\omega\left(\left[X_{1}, X_{2}\right]\right)+X_{1} \cdot \breve{f}_{2}-X_{2} \cdot \breve{f}_{1}+\Omega\left(X_{1}, X_{2}\right)+d \omega\left(X_{1}, X_{2}\right) ;\right. \\
& \left.\omega\left(\left[X_{1}, X_{2}\right]\right)\right) .
\end{aligned}
$$

On the other hand

$$
\begin{aligned}
\llbracket \boldsymbol{r}\left(X_{1}, \breve{f}_{1}\right) ; \boldsymbol{r}\left(X_{2}, \breve{f}_{2}\right) \rrbracket= & \left(\left\{\omega\left(X_{1}\right)+\breve{f}_{1}, \omega\left(X_{2}\right)+\breve{f}_{2}\right\} ;\right. \\
\left\{\omega\left(X_{1}\right)+\breve{f}_{1}, \omega\left(X_{2}\right)\right\}-\left\{\omega\left(X_{2}\right)+\breve{f}_{2}, \omega\left(X_{1}\right)\right\} & \left.-d \omega\left(d\left(\omega\left(X_{1}\right)+\breve{f}_{1}\right)^{\sharp}, d\left(\omega\left(X_{2}\right)+\breve{f}_{2}\right)^{\sharp}\right)\right) .
\end{aligned}
$$

These expressions are equal if and only if the following two equations are satisfied

$$
\begin{gathered}
\omega\left(\left[X_{1}, X_{2}\right]\right)+X_{1} \cdot \breve{f}_{2}-X_{1} \cdot \breve{f}_{2}+\Omega\left(X_{1}, X_{2}\right)+d \omega\left(X_{1}, X_{2}\right)= \\
\left\{\omega\left(X_{1}\right)+\breve{f}_{1}, \omega\left(X_{2}\right)+\breve{f}_{2}\right\}, \\
\omega\left(\left[X_{1}, X_{2}\right]\right)=\left\{\omega\left(X_{1}\right)+\breve{f}_{1}, \omega\left(X_{2}\right)\right\}-\left\{\omega\left(X_{2}\right)+\breve{f}_{2}, \omega\left(X_{1}\right)\right\} \\
-d \omega\left(d\left(\omega\left(X_{1}\right)+\breve{f}_{1}\right)^{\sharp}, d\left(\omega\left(X_{2}\right)+\breve{f}_{2}\right)^{\sharp}\right) .
\end{gathered}
$$

By using (3.4) and $\left(\Lambda^{\sharp} \otimes \Lambda^{\sharp}\right)(\Omega)=-\Lambda$ we can rewrite the left hand side of (3.5) as

$$
\begin{gathered}
X_{1} \cdot\left(\omega\left(X_{2}\right)+\breve{f}_{2}\right)-X_{2} \cdot\left(\omega\left(X_{1}\right)+\breve{f}_{1}\right)+\Omega\left(X_{1}, X_{2}\right)= \\
=i_{d\left(\omega\left(X_{1}\right)+\breve{f}_{1}\right)^{\sharp}+\omega\left(X_{1}\right) E} d\left(\omega\left(X_{2}\right)+\breve{f}_{2}\right)-i_{d\left(\omega\left(X_{2}\right)+\breve{f}_{2}\right)^{\sharp}+\omega\left(X_{2}\right) E} d\left(\omega\left(X_{1}\right)+\breve{f}_{1}\right) \\
+\Omega\left(d\left(\omega\left(X_{1}\right)+\breve{f}_{1}\right)^{\sharp}, d\left(\omega\left(X_{2}\right)+\breve{f}_{2}\right)^{\sharp}\right)= \\
=\left\{\omega\left(X_{1}\right)+\breve{f}_{1}, \omega\left(X_{2}\right)+\breve{f}_{2}\right\}-\left\{\omega\left(X_{2}\right)+\breve{f}_{2}, \omega\left(X_{1}\right)+\breve{f}_{1}\right\} \\
-\left\{\omega\left(X_{1}\right)+\breve{f}_{1}, \omega\left(X_{2}\right)+\breve{f}_{2}\right\}= \\
=\left\{\omega\left(X_{1}\right)+\breve{f}_{1}, \omega\left(X_{2}\right)+\breve{f}_{2}\right\} .
\end{gathered}
$$


Similarly, by using $i_{E} d(\omega(X))=d \omega(E, X)$, we can rewrite the left hand side of $(3.6)$ as

$$
\begin{aligned}
& X_{1} \cdot\left(\omega\left(X_{2}\right)\right)-X_{2} \cdot\left(\omega\left(X_{1}\right)\right)-d \omega\left(X_{1}, X_{2}\right)= \\
& =i_{d\left(\omega\left(X_{1}\right)+\breve{f}_{1}\right)^{\sharp}+\omega\left(X_{1}\right) E} d\left(\omega\left(X_{2}\right)\right)-i_{d\left(\omega\left(X_{2}\right)+\breve{f}_{2}\right)^{\sharp}+\omega\left(X_{2}\right) E} d\left(\omega\left(X_{1}\right)\right) \\
& \quad-d \omega\left(X_{1}, X_{2}\right)= \\
& =\left\{\omega\left(X_{1}\right)+\breve{f}_{1}, \omega\left(X_{2}\right)\right\}-\left\{\omega\left(X_{2}\right)+\breve{f}_{2}, \omega\left(X_{1}\right)\right\} \\
& \quad-d \omega\left(X_{1}-\omega\left(X_{1}\right) E, X_{2}-\omega\left(X_{2}\right) E\right) \\
& =\left\{\omega\left(X_{1}\right)+\breve{f}_{1}, \omega\left(X_{2}\right)\right\}-\left\{\omega\left(X_{2}\right)+\breve{f}_{2}, \omega\left(X_{1}\right)\right\} \\
& -d \omega\left(d\left(\omega\left(X_{1}\right)+\breve{f}_{1}\right)^{\sharp}, d\left(\omega\left(X_{2}\right)+\breve{f}_{2}\right)^{\sharp}\right)
\end{aligned}
$$

which proves that (3.3) is a Lie algebra morphism.

Finally, it is easy to see that $(\boldsymbol{r} \circ \boldsymbol{s})(f, h)=(f, h)$ for all $(f, h) \in$ $\operatorname{LGen}(\omega, \Omega)$.

\section{EXAMPLES}

In this section we recall results obtained for structures of the classical phase space. These results were the motivation of the paper.

We assume classical space-time to be an oriented and time oriented 4-dimensional manifold $\boldsymbol{E}$ equipped with a Lorentzian metric $g$, with signature $(1,3)$, [7]. We denote by $\left(x^{\lambda}\right)=\left(x^{0}, x^{i}\right), \lambda=0,1,2,3$, local coordinates on $\boldsymbol{E}$ such that $\partial_{0}$ is time-like and $\partial_{i}$ are space-like. A motion is defined to be a 1-dimensional time-like submanifold of space-time. We define the classical (Einsteinian) phase space to be the open subspace $\mathcal{J}_{1} \boldsymbol{E} \subset J_{1}(\boldsymbol{E}, 1)$ consisting of all 1-jets (1st order contact elements) of motions. So elements of $\mathcal{J}_{1 x} \boldsymbol{E}$ are classes of nonparametrized curves which have in a point $x \in \boldsymbol{E}$ the same tangent line lying inside the light cone. $\pi_{0}^{1}: \mathcal{J}_{1} \boldsymbol{E} \rightarrow \boldsymbol{E}$ is a fibred manifold but not an affine bundle! We have the induced coordinate chart $\left(x^{\lambda}, x_{0}^{i}\right)$.

The metric $g$ gives naturally the unscaled horizontal time form

$$
\widehat{\tau}: \mathcal{J}_{1} \boldsymbol{E} \rightarrow T^{*} \boldsymbol{E}, \quad \widehat{\tau}=\widehat{\tau}_{\lambda} d x^{\lambda} .
$$

\subsection{Infinitesimal symmetries of the gravitational contact struc-} ture. The pair $\left(-\widehat{\tau}, \Omega^{\mathfrak{g}}\right)$, where

$$
\Omega^{\mathfrak{g}}=-d \widehat{\tau}: \mathcal{J}_{1} \boldsymbol{E} \rightarrow \bigwedge^{2} T^{*} \mathcal{J}_{1} \boldsymbol{E}
$$

is the contact (gravitational) regular structure on $\mathcal{J}_{1} \boldsymbol{E}$. The dual Jacobi structure is given by a pair $\left(-\widehat{\gamma}^{\mathfrak{g}}, \Lambda^{\mathfrak{g}}\right)$, where $\widehat{\gamma}^{\mathfrak{g}}$ and $\Lambda^{\mathfrak{g}}$ are naturally given by the metric field (for details see [7]). 
By Corollary 2.8 infinitesimal symmetries of the gravitational contact phase structure are Hamilton-Jacobi lifts of conserved functions, i.e. they are of the type $X=d f^{\sharp}+f \widehat{\gamma}^{\mathfrak{g}}$, where $\widehat{\gamma}^{\mathfrak{g}} \cdot f=0$ and, moreover, $f=\widehat{\tau}(X)=\widehat{\tau}(\underline{X})$. Here $\underline{X}=T \pi_{0}^{1}(X): \mathcal{J}_{1} \boldsymbol{E} \rightarrow T \boldsymbol{E}$ is a generalized vector field in the terminology of [14]. So infinitesimal symmetries are of the type

$$
X=d(\widehat{\tau}(\underline{X}))^{\sharp}+\widehat{\tau}(\underline{X}) \widehat{\gamma}^{\mathfrak{g}},
$$

where the following conditions are satisfied

1. (Projectability) The Hamilton-Jacobi lift (4.1) projects on $\underline{X}$.

2. (Conservation) $\widehat{\tau}(\underline{X})$ is conserved, i.e. $\gamma^{\mathfrak{g}} \cdot(\widehat{\tau}(\underline{X}))=0$.

The following results were proved in [3].

Lemma 4.1. A symmetric $k$-vector field $\stackrel{k}{K}, k \geq 1$, on $\boldsymbol{E}$ admits the generalized vector field satisfying the projectability condition. Such generalized vector fields are given by

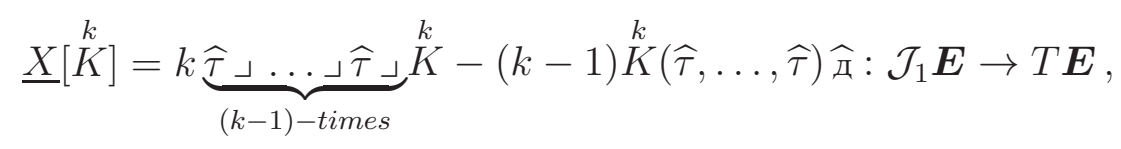

where $\widehat{\mathrm{A}}=T \pi_{0}^{1}\left(\widehat{\gamma}^{\mathfrak{g}}\right)$. Then we obtain the induced phase function

$$
\widehat{\tau}(\underline{X}[\stackrel{k}{K}])=\stackrel{k}{K}(\widehat{\tau})=\stackrel{k}{K}(\widehat{\tau}, \ldots, \widehat{\tau})=\stackrel{k}{K}^{\lambda_{1} \ldots \lambda_{k}} \widehat{\tau}_{\lambda_{1}} \ldots \widehat{\tau}_{\lambda_{k}}
$$

Lemma 4.2. Let $\stackrel{0}{K}$ be a space-time function. Then $\widehat{\gamma}^{\mathfrak{g}} \cdot \stackrel{0}{K}=0$ if and only if $\stackrel{0}{K}$ is a constant. The phase function $\stackrel{k}{K}(\widehat{\tau}), k \geq 1$, is conserved with respect to the gravitational Reeb vector field, i.e. $\widehat{\gamma}^{\mathfrak{g}} \cdot \stackrel{k}{K}(\widehat{\tau})=0$, if and only if $\stackrel{k}{K}$ is a Killing k-vector field.

Theorem 4.3. The Hamilton-Jacobi lift of a phase function

$$
K=\stackrel{0}{K}+\sum_{k \geq 1} \stackrel{k}{K}(\widehat{\tau})
$$

is an infinitesimal symmetry of the gravitational contact phase structure $\left(-\widehat{\tau}, \Omega^{\mathfrak{g}}\right)$ if and only if $\stackrel{0}{K}$ is a constant and $\stackrel{k}{K}, k \geq 1$, are Killing $k$-vector fields. Moreover, for $k=1$ the corresponding infinitesimal symmetry coincides with the jet flow lift $\mathcal{J}_{1} \stackrel{1}{K}$ and is projectable on space-time. For $k \geq 2$ the corresponding infinitesimal symmetry is hidden. 
Remark 4.1. It is very well known that Killing multivector fields generates on $T^{*} \boldsymbol{E}$ functions constant of motion (functions constant on lifts of geodesic curves) (see, for instance, 15]). In [4 it was proved that if we consider the mapping $-\widehat{\tau}: \mathcal{J}_{1} \boldsymbol{E} \rightarrow T^{*} \boldsymbol{E}$, then a conserved phase function of type (4.2) is obtained as the pull-back $K=-\widehat{\tau}^{*}(\tilde{K})$ of a function $\tilde{K}$ constant of motion.

\subsection{Infinitesimal symmetries of the total almost-cosymplectic-} contact phase structure. Let us assume an electromagnetic (Maxwell) field which is a closed 2-form $\widehat{F}: \boldsymbol{E} \rightarrow \wedge^{2} T^{*} \boldsymbol{E}$. Then we can consider the total phase 2 -form

$$
\Omega^{\mathfrak{j}}=: \Omega^{\mathfrak{g}}+\Omega^{\mathfrak{e}}=-d \widehat{\tau}+\frac{1}{2} \widehat{F}
$$

and the pair $\left(-\widehat{\tau}, \Omega^{j}\right)$ turns out to be an almost-cosymplectic-contact structure of the phase space, i.e. $\Omega^{j}$ is closed and $\widehat{\tau} \wedge \Omega^{j} \wedge \Omega^{j} \wedge \Omega^{j}$ is a volume form.

The dual almost-coPoisson-Jacobi pair is then given by the total Reeb vector field $\widehat{\gamma}^{\mathfrak{j}}=\widehat{\gamma}^{\mathfrak{g}}+\widehat{\gamma}^{\mathfrak{e}}$ and the total phase 2-vector $\Lambda^{\mathfrak{j}}=\Lambda^{\mathfrak{g}}+\Lambda^{\mathfrak{e}}$, where $\widehat{\gamma}^{\mathfrak{e}}$ and $\Lambda^{\mathfrak{e}}$ are $\boldsymbol{E}$-vertical given by $g$ and $\widehat{F}$, see [7].

In [4] it was proved that all phase infinitesimal symmetries of the total phase structure are vector fields of the type

$$
X=d(\widehat{\tau}(\underline{X})+\breve{f})^{\sharp j}+\widehat{\tau}(\underline{X}) \widehat{\gamma}^{j}
$$

where $\underline{X}$ is a generalized vector field and $\breve{f} \in C^{\infty}(\boldsymbol{E})$ such that:

1) $d \breve{f}=\underline{X}\lrcorner \widehat{F}$

2) (Projectability) The vector field $X$ projects on $\underline{X}$;

3) (Conservation) $\widehat{\gamma}^{j} \cdot(\widehat{\tau}(\underline{X})+\breve{f})=0$.

The projectability condition is the same as in the case of the contact gravitational structure which follows from the fact that the fields $\widehat{\gamma}^{\mathfrak{e}}$ and $\Lambda^{\mathfrak{e}}$ are $\boldsymbol{E}$-vertical. So it is sufficient to describe conditions that the function (4.2), where $\breve{f}=\stackrel{0}{K}$, is conserved.

Theorem 4.4. 4] A phase function (4.2) is conserved, i.e. $\widehat{\gamma}^{\mathrm{j}} \cdot K=0$, if and only if

$$
\begin{gathered}
g^{\rho \lambda} \partial_{\rho} \stackrel{0}{K}+\stackrel{1}{K^{\rho}} \widehat{F}_{\rho}^{\lambda}=0, \\
\nabla^{\left(\lambda_{1}\right.} \stackrel{k}{K}^{\left.\lambda_{2} \ldots \lambda_{k+1}\right)}+(k+1) \stackrel{k+1}{K}{ }^{\rho\left(\lambda_{1} \ldots \lambda_{k}\right.} \widehat{F}^{\left.\lambda_{k+1}\right)}{ }_{\rho}=0,
\end{gathered}
$$

for $k \geq 1$. 
Corollary 4.5. Let us assume a special phase function $K=\stackrel{0}{K}+\stackrel{1}{K}(\widehat{\tau})$. Then the conditions (4.3) and (4.4) are reduced to

$$
\partial_{\rho} \stackrel{0}{K}-\stackrel{1}{K} \sigma \widehat{F}_{\sigma \rho}=0, \quad \nabla^{\left(\lambda_{1}\right.} \stackrel{1}{K^{\left.\lambda_{2}\right)}}=0
$$

and we obtain the result of $[9]$, i.e. $\stackrel{1}{K}$ is a Killing vector field and $\stackrel{0}{K}$ and $\stackrel{1}{K}$ are related by the formula $d \stackrel{0}{K}=\stackrel{1}{K}\lrcorner \widehat{F}$. Moreover, the corresponding infinitesimal symmetry is the jet flow lift $\mathcal{J}_{1} \stackrel{1}{K}$ which projects on $\stackrel{1}{K}$.

Remark 4.2. Let us assume a phase function $K=\stackrel{k}{K}(\widehat{\tau}), \quad k \geq 2$. Then the condition (4.4) gives

$$
\nabla^{\left(\lambda_{1}\right.} \stackrel{k}{K}{ }^{\left.\lambda_{2} \ldots \lambda_{k+1}\right)}=0, \quad \stackrel{k}{K}{ }^{\rho\left(\lambda_{1} \ldots \lambda_{k-1}\right.} \widehat{F}^{\left.\lambda_{k}\right)}{ }_{\rho}=0
$$

and we obtain that $\stackrel{k}{K}$ is a Killing-Maxwell $k$-vector field. But the corresponding lift has to satisfy also the condition 1) which is of the form $\underline{X}[K]\lrcorner \widehat{F}=0$. This condition implies $\widehat{\nexists}\lrcorner \widehat{F}=0$, which implies $\widehat{F} \equiv 0$ and the structure is reduced to the gravitational case. This implies that there are no non-projectable (hidden) infinitesimal symmetries generated by Killing-Maxwell $k$-vector fields for $k \geq 2$.

So all infinitesimal symmetries of $\left(-\widehat{\tau}, \Omega^{\mathfrak{j}}\right)$ are projectable and can be generated by pairs $(\underline{X}, \breve{f})$ of Killing vector fields and spacetime functions such that $d \breve{f}=\underline{X}\lrcorner \widehat{F}$. Such pairs are sections of the Lie algebroid $T \boldsymbol{E} \oplus \mathbb{R} \rightarrow \boldsymbol{E}$ with the bracket $\llbracket ; \rrbracket_{\widehat{F}}$ (see Section 3 and [9]). The sections of the Lie algebroid described in Section 3 are obtained as the 1-jet flow lifts of $\underline{X}$ and the pull-backs of $\breve{f}$.

\section{REFERENCES}

[1] M. de Leon, G.M. Tuynman: A universal model for cosymplectic manifolds, Journal of Geometry and Physics 20 (1996), 77-86, DOI: 10.1016/03930440(96)00047-2.

[2] J. Janyška: Special phase functions and phase infinitesimal symmetries in classical general relativity, AIP Conf. Proc. 1460, XX Internat. Fall Workshop on Geometry and Physics, 135-140, DOI: 10.1063/1.4733369.

[3] J. Janyška: Hidden symmetries of the gravitational contact structure of the classical phase space of general relativistic test particle, Arch. Math. (Brno) 50(5) (2014), 297-316, DOI: 10.5817/AM2014-5-297.

[4] J. Janyška: Relations between constants of motion and conserved functions, Arch. Math.(Brno) 51 (2015), 297-313, DOI: 10.5817/AM2015-5-297.

[5] J. Janyška: On Lie algebras of generators of infinitesimal symmetries of almost-cosymplectic-contact structures, to appear in Arch. Math. (Brno), arXiv: 1610.06714. 
[6] J. Janyška, M. Modugno: Graded Lie algebra of Hermitian tangent valued forms, J. Math. Pures Appl. 85 (2006), 687-697, DOI: 10.1016/j.matpur. 2005.11.004.

[7] J. Janyška, M. Modugno: Geometric Structures of the Classical General Relativistic Phase Space, Int. J. Geom. Methods Mod. Phys. 5 (2008), 699-754, DOI: $10.1142 / \mathrm{S} 021988780800303 X$.

[8] J. Janyška, M. Modugno: Generalized geometrical structures of odd dimensional manifolds, J. Math. Pures Appl. (9) 91 (2009), 211-232, DOI: 10.1016/j.matpur.2008.09.007.

[9] J. Janyška, R. Vitolo: On the characterization of infinitesimal symmetries of the relativistic phase space, J. Phys. A: Math. Theor. 45 (2012), 485205, DOI: 10.1088/1751-8113/45/48/485205.

[10] A. A. Kirillov: Local Lie algebras, Russian Math. Surveys 31 (1976), 55-76.

[11] I. Koláŕ, P. W. Michor, J. Slovák: Natural Operations in Differential Geometry, Springer 1993.

[12] A. Lichnerowicz: Les varietés de Jacobi et leurs algèbres de Lie associées, J. Math. pures et appl. 57 (1978), 453-488.

[13] K. Mackenzie: General Theory of Lie Groupoids and Lie Algebroids, London Mathematical Society, Lecture Note Series 213, Cambridge University Press 2005.

[14] P. Olver: Applications of Lie groups to differential equations, Graduate Texts in Mathematics 107, Springer 1986.

[15] P. Sommers: On Killing tensors and constant of motions, J. Math. Phys. 14 (1973), 787790.

[16] I. Vaisman: Lectures on the Geometry of Poisson Manifolds, Birkhäuser Verlag, Basel-Boston-Berlin 1994.

Department of Mathematics and Statistics, Masaryk University

Kotlářská 2, 61137 Brno, Czech Republic

E-MAIL:JANYSKA@MATH.MUNI.CZ 